\title{
FAILURE RATE MEASUREMENT ON SILICON DIODES REVERSE POLARIZED AT HIGH TEMPERATURE
}

\author{
D. Osorno, E. Sanchis-Kilders, E. Maset, D. Gilabert, A. Ferreres, J. Jordán, V. Esteve, J.L. Gasent-Blesa \\ Dpt. IE / ETSE, Universidad de Valencia, Avd. de la Universitat s/n, 46100 Burjassot, Spain, daniel.osorno@uv.es
}

\begin{abstract}
This paper calculates the failure rate on reversed polarized silicon diodes with the aim to justify, experimentally, the rules of the European Space Agency (ESA) which are referred to the component life's extension, the reliability increase and the end of life performance enhancement, by using oversized devices (derating rules).

In order to verify the derating rules, 80 silicon diodes are used, which are reverse polarized in a high temperature environment. The diodes are divided in 4 groups of 20 diodes, applying a different voltage to each group, in order to relate the failure rate to the applied derating rule. The experiment described in this paper is developed using a temperature accelerated test to check the leakage current in reverse polarization (HTRB - High Temperature Reverse Bias), with the purpose of obtaining results applying an acceleration factor in order to reduce the test duration.

By using a thermal model of the whole system and the equations that describe the reverse polarized diode behaviour, it is possible to stress the 80 diodes up to very high temperature avoiding the runaway effect.

Finally, the failure rate is calculated and a revision of the derating rules are proposed by using the experimental result obtained.
\end{abstract}

\section{INTRODUCTION}

ESA (European Space Agency) uses rules to extend the life of the devices, thus guarantee the proper operation for the duration of the mission [1]. This practice, which oversizes the devices and makes them work below their maximum ratings, is known as "derating".

But these rules, mandatory in space missions, increase the economic cost, the weight and the size of the selected devices to use for the design. These rules also reduces the list of a available devices for design. Thus complicating the suitable selection of these devices (which are not always available for space).

M. de Economía y Competitividad of Gobierno de España and FEDER; Ref. ESP2013-47349-C6-5-R y ESP2104-56169-C6-4-R.

Conselleria d'Educació, Formació i Ocupació, Generalitat Valenciana; Ref. PROMETEO/2012/044.
In the end, the selection of the needed devices becomes a difficult task and increases the cost of the development. Of course this assures a higher reliability.

For example, the derating rules established by ESA for a Schottky diode define maximum values not to be surpassed and are as follows:

- $75 \%$ of maximum forward current,

- $75 \%$ of maximum reverse voltage,

- $50 \%$ of maximum dissipated power and

- a maximum junction temperature of $110^{\circ} \mathrm{C}$ or $40^{\circ} \mathrm{C}$ below the manufacturer's maximum rating, whichever is lower.

Thus, in the case of the reverse voltage, the maximum value permitted under derating conditions would be $75 \%$ of the maximum reverse voltage specified in the datasheet of the manufacturer.

Additionally, NASA (National Aeronautics and Space Administration) also proposes derating rules for devices, although they are different, in some cases, from the rules proposed by ESA.

For example, the rules from NASA of 2003 [2], for the same Schotkky diode of the last example, establishes the following deratings:

- $50 \%$ of maximum forward current,

- $70 \%$ of peak inverse voltage (PIV),

- $50 \%$ of maximum peak current,

- $80 \%$ of maximum junction temperature (does not exceed $125^{\circ} \mathrm{C}$ or $40^{\circ} \mathrm{C}$ below the manufacturer's maximum rating, whichever is lower).

And some years before, in 1991, NASA had published derating rules [3] that resembled those currently used by ESA, but different to the used nowadays. In the example of the Schottky diode, the former compulsory derating asked by NASA then was:

- $75 \%$ of maximum reverse voltage,

- $50 \%$ of maximum dissipated power and

- a maximum junction temperature of $110^{\circ} \mathrm{C}$. 
Other references [4] also propose that the failure rate is related to the stress and follows the $5^{\text {th }}$ power law, which says, for example, that an increase of a $15 \%$ of the applied voltage to the device, implies doubling the failure rate.

Summarising, the derating rules proposed by ESA and NASA, and other laws also related to derating, applied to electronic devices, are all based on mathematical models and also in the practical experience. However, it is possible to consider that a 10 year long mission should have a different requirement than a mission that is only 6 months long, thus, the derating rules should be different. Also, when both rules (ESA's and NASA's) are compared, some differences are detected that could mean that the reliability of electronic devices is still not fully understood.

A more in depth research is therefore needed, to better understand the reliability of semiconductor devices, together with experimental results to verify this research. The following question should be answered: are derating the rules applicable to any mission? and, could these rules be updated with new results and in order to optimise cost, mass and volume of space missions? [5] [6]

The experimental results obtained that correspond to the failure rate as a function of the applied derating can be used to justify and/or update the existing derating rules applied in space missions.

\section{EXPERIMENT DEVELOPMENT}

This paper follows these steps:
A) Selection of the device under test.
B) Selection of the variable to analyse.
C) Experimental setup.
D) Auxiliary components.
E) Proposed thermal model.
F) Analysis of the acceleration factor.

The first task to do consist in selecting the device and the variable to test. The next task is to propose an experimental setup. Then all additionally needed components of the setup will be identified. Finally, the thermal model and the acceleration factor will be calculated in order to predict the results of the experiment.

These steps should guarantee positive experimental results showing the failure rate of the devices.

\subsection{Selection of the device under test}

In order to simplify the experiment, a diode is considered as a device under test (DUT), because other different devices, like transistors imply additional complexity. The final selection of the DUT is based on the diode list found in the EPPL (European Preferred Parts List) [7].

A commercial equivalent for each diode of this list is identified, and then, a selection is made keeping in mind, availability, electric characteristics, package and price of this equivalent diode (see Tab. 1).

Table 1. Diode selection

\begin{tabular}{|c|c|c|c|}
\hline EPPL & Commercial & Package & Price (€) \\
\hline 1N6642U & 1N6642D2A & $\overline{\text { DLCC2 }}$ & $\overline{24}$ \\
\hline BYW81-200 & DPG15I200PA & TO-220AC & 1.23 \\
\hline BYW81-200 & BYV79E-20 & TO-220AC & 0.98 \\
\hline BYW52-200 & BYW51-200G & TO-220AB & 0.76 \\
\hline BYW52-200 & $\mathrm{NO}$ & & \\
\hline 1N5811US & 1N5811 & PTH & \\
\hline 1N5806US & 1N5806 & DO-204AP & 4.45 \\
\hline 1N5416US & $\mathrm{NO}$ & & \\
\hline 1N5417US & 1N5417 & PTH & \\
\hline 1N5418US & 1N5418 & PTH & \\
\hline 1N5420US & 1N5420 & PTH & \\
\hline 1N54189UR-1 & $\mathrm{NO}$ & & \\
\hline STPS1045 & STPS1045B-TR & D-PAK & 0.47 \\
\hline STPS6045 & STPS6045CW & TO-247 & 3.4 \\
\hline STPS40100 & NO & TO-220AB & 0.76 \\
\hline 1N5822U & 1N5822 & PTH & \\
\hline 1N5615 & $\mathrm{NO}$ & & \\
\hline 1N5617 & 1N5617 & PTH & \\
\hline 1N5619 & 1N5619 & PTH & \\
\hline $1 \mathrm{~N} 5623$ & $\mathrm{NO}$ & & \\
\hline STPS20100 & $\mathrm{NO}$ & & \\
\hline $1 \mathrm{~N} 5416$ & NO & & \\
\hline 1N5417 & $\mathrm{NO}$ & & \\
\hline 1N5418 & NO & & \\
\hline $1 \mathrm{~N} 5420$ & NO & & \\
\hline 1N5806U & 1N5806D2A & TO-220AB & 14.76 \\
\hline 1N5819U & 1N5819HW-7-F & TO-220AB & 0.24 \\
\hline 1N5614 & $\mathrm{NO}$ & & \\
\hline 1N5616 & NO & & \\
\hline $1 \mathrm{~N} 5618$ & 1N5618 & PTH & \\
\hline $1 \mathrm{~N} 5550$ & NO & & \\
\hline 1N5552 & $\mathrm{NO}$ & & \\
\hline 1N5554 & NO & & \\
\hline BAS70 & BAS70-05 & SOT-23 & 0.03 \\
\hline
\end{tabular}

After discarding the diodes which have no commercial equivalent or have a different package than SMD (in order to solder the device on a PCB to a copper plane), the diode STPS1045 is selected as the best option for this test, due to its size, package and price. Additionally, this diode is an European low voltage Schottky, which is commonly used in space missions in output rectifiers of $3.3 \mathrm{~V}$ and $5 \mathrm{~V}$.

This diode has a maximum reverse voltage of $45 \mathrm{~V}$, and a reverse leakage current of $1.8 \mathrm{~mA}$ at $100^{\circ} \mathrm{C}(45 \mathrm{~V})$.

\subsection{Selection of the variable to analyse}

ESA's diode derating rules consider: direct current, reverse voltage, dissipated power and junction temperature.

In the first analysis, the dissipated power and the junction temperature won't be studied, because these characteristics can be calculated as a function of the direct current and/or reverse voltage. 


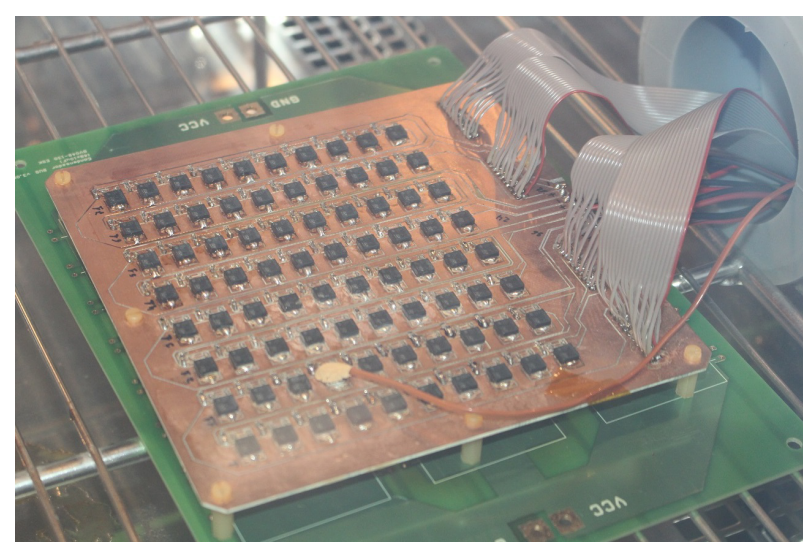

Figure 1. Setup

Finally, the reverse voltage $\left(V_{R}\right)$ is chosen as a characteristic to analyse. The reverse current will be measured using a shunt resistor connected in series to the DUT and independently from the other diodes. Additionally, the influence of the self-heating effect is less important in reverse polarization than in direct polarization.

\subsection{Experimental setup}

Once the diode and the characteristic to analyse is chosen, it is proposed to carry out an accelerated test increasing the temperature [8], [9] y [10], also known as an HTRB (High Temperature Reverse Bias) test. The derating rules will be applied as reverse biased diode, limiting the maximum reverse voltage and it will be studied how this extends life of the devices.

In order to guarantee the thermal uniformity of all DUT, they must be soldered to the copper plane of the PCB as close to each other as possible, and placed inside a climatic chamber, which will control the ambient temperature surrounding the devices and all its setup (see Fig. 1).

During the test continuous reverse voltage stress will be applied to the diodes reverse and temperature will be kept constant.

The total population of diodes used in this experiment is 80 , which will be divided in 4 groups of 20 diodes and each group is reverse biased with a different voltage $\left(V_{R}\right)$. In order to obtain results, that could show which is the most convenient derating to apply, the following reverse voltages $\left(V_{R}\right)$ are proposed:

$$
\begin{aligned}
& \text { - } V_{R}=100 \%(45 \mathrm{~V}) \\
& \text { - } V_{R} \approx 80 \%(36 \mathrm{~V}) \\
& \text { - } V_{R} \approx 50 \%(24 \mathrm{~V}) \\
& \text { - } V_{R} \approx 30 \%(12 \mathrm{~V})
\end{aligned}
$$

\subsection{Auxiliary components}

To measure the reverse leakage current $\left(I_{R}\right)$ that appears when the diode is reverse polarized, which is the variable

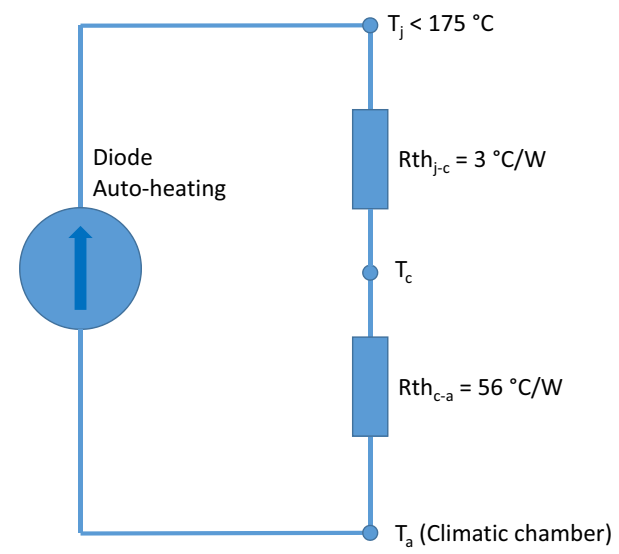

Figure 2. Thermal diagram

to analyse, a shunt resistors will be used in series with each diode.

The value of this shunt resistor must maximize the measurement resolution and, at the same time, the voltage drop should not interfere in the experiment $\left(V_{R_{\text {shunt }}}<2 \%\right.$ of the voltage $V_{R}$ ), then finally, the shunt resistor's value selected are:

$$
\begin{aligned}
& \text { - } V_{R}=45 \mathrm{~V} \Rightarrow 75 \Omega \\
& \text { - } V_{R}=35 \mathrm{~V} \Rightarrow 108.33 \Omega \\
& \text { - } V_{R}=24 \mathrm{~V} \Rightarrow 390 \Omega \\
& \text { - } V_{R}=12 \mathrm{~V} \Rightarrow 390 \Omega
\end{aligned}
$$

\subsection{Proposed thermal model}

With the aim of guaranteeing that the DUT does not reach the maximum junction temperature and/or thermal runaway due to self-heating during the experiment, the operating temperature for the HTRB must be chosen using an appropriate thermal model of the setup.

To do this, the thermal resistance from junction-to-case $\left(R t h_{j-c}=3{ }^{\circ} \mathrm{C} / \mathrm{W}\right)$ and from case-to-ambient $\left(R t h_{c-a}=56{ }^{\circ} \mathrm{C} / \mathrm{W}\right)$ is obtained from the datasheet of the DUT. This last parameter depends on the copper area which the DUT is soldered to.

These constants are included into the thermal diagram of the used setup, obtaining thus the thermal model (see Fig. 2).

On the other hand, from the $I_{R}$ vs. $V_{R}$ curve of the datasheet of the DUT (see Fig. 3), the $I_{R}$ values can be obtained for different points of $T_{j}\left(25^{\circ} \mathrm{C}, 50^{\circ} \mathrm{C}, 75^{\circ} \mathrm{C}\right.$, $100^{\circ} \mathrm{C}, 125^{\circ} \mathrm{C}$ and $150^{\circ} \mathrm{C}$ ) for the four used values of $V_{R}(45 \mathrm{~V}, 35 \mathrm{~V}, 24 \mathrm{~V}$ and $12 \mathrm{~V}$ ) (see Fig. 4). Next, with these values, it is possible to obtain the equations of the regression curves given by any curve fitting software, by using $5^{\text {th }}$ order (see Fig. 4 and equations 1, 2, 3 y 4), for each of the four reverse voltages of this test.

With these four equations, being $V_{R}=45 \mathrm{~V}$ the voltage with more self-heating influence in the junction temperature, a recursive simulation is carried out, using 


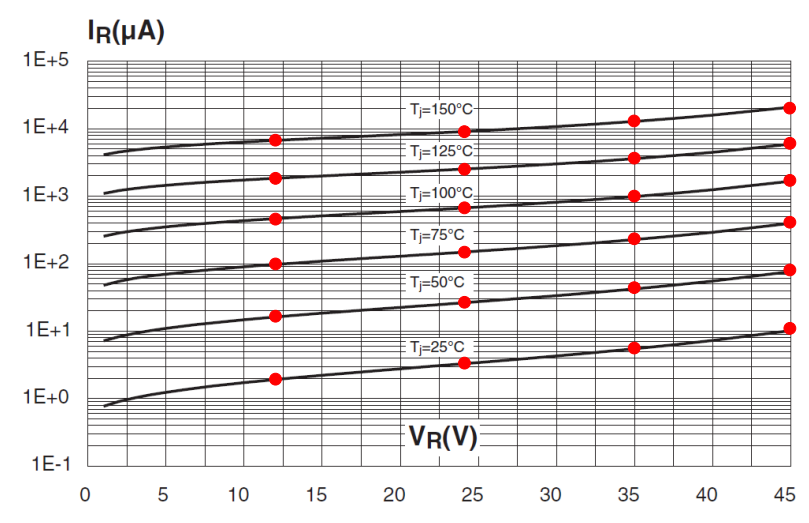

Figure 3. Relationship $I_{R}$ vs. $V_{R}$ found in the DUT's datasheet.

the equation of the dissipated power of the diode (Eq. 5 ) and the expression that relates $T_{j}$ with this dissipated power (Eq. 6) in order to verify the diode does not thermally runaway. In this case we can determine the maximum operating temperature to stress the diodes, before thermal runaway occurs.

$$
\begin{aligned}
& V_{R}=45 \mathrm{~V} \Rightarrow I_{R}(\mathrm{~mA})= \\
& =3.7248 \cdot 10^{-06} \cdot T_{j}{ }^{5}-1.2997 \cdot 10^{-03} \cdot T_{j}{ }^{4}+ \\
& +1.8231 \cdot 10^{-01} \cdot T_{j}{ }^{3}-1.2068 \cdot 10^{+01} \cdot T_{j}{ }^{2}+ \\
& +3.6960 \cdot 10^{+02} \cdot T_{j}-4.06500 \cdot 10^{+03} \\
& V_{R}=35 \mathrm{~V} \Rightarrow I_{R}(\mathrm{~mA})= \\
& =1.7916 \cdot 10^{-06} T_{j}{ }^{5}-6.1835 \cdot 10^{-04} \cdot T_{j}{ }^{4}+ \\
& +8.7004 \cdot 10^{-02} \cdot T_{j}{ }^{3}-5.8015 \cdot 10^{+00} \cdot T_{j}{ }^{2}+ \\
& +1.7912 \cdot 10^{+02} \cdot T_{j}-1.9820 \cdot 10^{+03} \\
& V_{R}=24 \mathrm{~V} \Rightarrow I_{R}(\mathrm{~mA})= \\
& =2.6767 \cdot 10^{-06} \cdot T_{j}{ }^{5}-9.6205 \cdot 10^{-04} \cdot T_{j}{ }^{4}+ \\
& +1.3530 \cdot 10^{-01} \cdot T_{j}{ }^{3}-8.9491 \cdot 10^{+00} \cdot T_{j}{ }^{2}+ \\
& +2.7331 \cdot 10^{+02} \cdot T_{j}-3.0008 \cdot 10^{+03} \\
& V_{R}=12 \mathrm{~V} \Rightarrow I_{R}\left(\mathrm{~mA}{ }^{+}=\right. \\
& =5.8795 \cdot 10^{-07} \cdot T_{j}{ }^{5}-1.7472 \cdot 10^{-04} \cdot T_{j}{ }^{4}+ \\
& +2.2493 \cdot 10^{-02} \cdot T_{j}{ }^{3}-1.4188 \cdot 10^{+00} \cdot T_{j}{ }^{2}+ \\
& +4.2512 \cdot 10^{+01} \cdot T_{j}-4.6400 \cdot 10^{+02} \\
& \quad P_{D}(W)=I_{R} \cdot V_{R}-R_{s h u n t} \cdot I_{R}{ }^{2}
\end{aligned}
$$

where,

- $P_{D}$ is the dissipated power in $\mathrm{W}$

- $I_{R}$ is the reverse leakage current in $\mathrm{A}$

- $V_{R}$ is the reverse voltage in $\mathrm{V}$

- $R_{\text {shunt }}$ is the value of the shunt resistor in $\Omega$

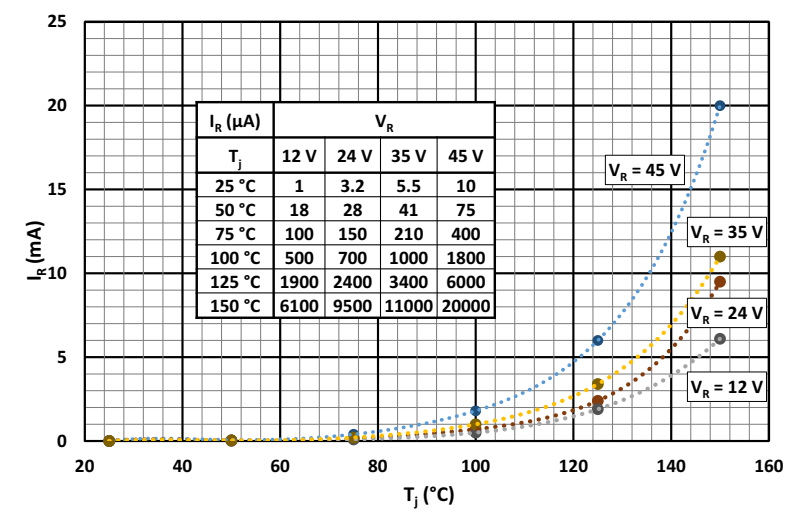

Figure 4. Values extraction of $I_{R}$ as a function of $T_{j}$ and $V_{R}$, and adjustment to $5^{\text {th }}$ polynomial order.

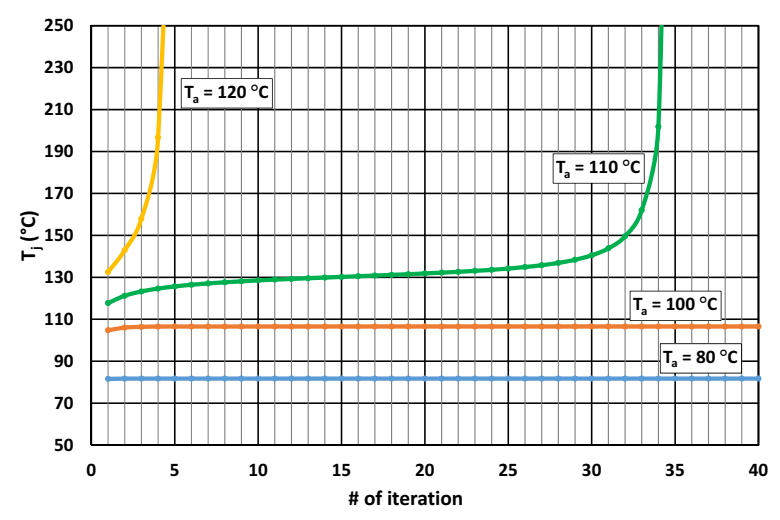

Figure 5. Results of the recursive simulation where a thermal runaway is observed for $T_{j}=110^{\circ} \mathrm{C}$ and $T_{j}=$ $120^{\circ} \mathrm{C}$

$$
T_{j}\left({ }^{\circ} \mathrm{C}\right)=T_{a}+\left(R t h_{j-c}+R t h_{c-a}\right) \cdot P_{D}
$$

where,

- $T_{j}$ is the junction temperatures expressed in ${ }^{\circ} \mathrm{C}$

- $T_{a}$ is the operating temperature expressed in ${ }^{\circ} \mathrm{C}$

- $R t h_{j-c}$ is the thermal resistance from junction-to-case expressed in $\mathrm{W} /{ }^{\circ} \mathrm{C}$

- $R t h_{c-a}$ is the thermal resistance from case-to-ambient expressed in $\mathrm{W} /{ }^{\circ} \mathrm{C}$

- $P_{D}$ is the dissipated power in $\mathrm{W}$.

The result given by the recursive simulation (see Fig. 5 ) is that, with an operating temperature of about $110^{\circ} \mathrm{C}$, diodes blocking $V_{R}=45 \mathrm{~V}$ reach the thermal runaway $\left(T_{j}>150^{\circ} \mathrm{C}\right)$ destroying the silicon junction of the semiconductor. Therefore, the temperature of the experiment is reduced by $10^{\circ} \mathrm{C}$ and so fixed at $100^{\circ} \mathrm{C}$, programming the climatic chamber used. 


\subsection{Analysis of the acceleration factor}

As said before, the experiment shown in this paper stressed the diodes after derating their limits [1], which makes more difficult to obtain a failure. To observe a failure an accelerated test will be done.

By using the Arrhenius' equation (Eq. 7) in order to calculate this acceleration factor, customized by NASA

(Eq. 8), where the constant of activation energy is $\left(E_{A}=0.2656 \mathrm{eV}\right)$ [11] and knowing the operating temperature of the experiment $\left(T_{a}=100{ }^{\circ} \mathrm{C}\right)$, the obtained acceleration factor is equal to 8 units. Por example, 1000 hours of this test are equivalent to 8000 hours of operation of the DUT (at $25^{\circ} \mathrm{C}$ ).

$$
A_{T}=\frac{\lambda_{T 1}}{\lambda_{T 2}}=e^{\left[\frac{E_{A}}{k}\left(\frac{1}{T_{1}}-\frac{1}{T_{2}}\right)\right]}
$$

where,

- $A_{T}$ acceleration factor

- $T_{1}$ y $T_{2}$ is the temperature 1 and 2 respectively in ${ }^{\circ} \mathrm{C}$

- $\lambda_{T 1}$ and $\lambda_{T 2}$ is the failure rate at temperature 1 and 2 respectively

- $E_{A}$ is the activation energy in $\mathrm{eV}$

- $k$ is the Boltzmann constant in $\mathrm{eV} /{ }^{\circ} \mathrm{C}$

$$
\pi_{T}=\frac{\lambda_{T_{j}}}{\lambda_{25^{\circ} \mathrm{C}}}=e^{\left[-3091\left(\frac{1}{T_{j}-273}-\frac{1}{298}\right)\right]}
$$

where,

- $\pi_{T}$ is the acceleration factor

- $\lambda_{T_{j}}$ is the failure rate at the operating temperature

- $\lambda_{25}{ }^{\circ} \mathrm{C}$ is the failure rate at $25^{\circ} \mathrm{C}$

- $T_{j}$ is the junction temperature of the semiconductor in $\mathrm{K}$

\section{RESULTS}

Until now, the results (see Fig. 6) show a quite stable behaviour of the reverse leakage current for the four derated diode groups. In a first transient stage, an increase of the reverse leakage current is observed until it reaches the steady-state after the adjustment of temperature of the climatic chamber up to $100^{\circ} \mathrm{C}$.

The standard calculation method to obtain the failure rate is shown in Eq. 9.

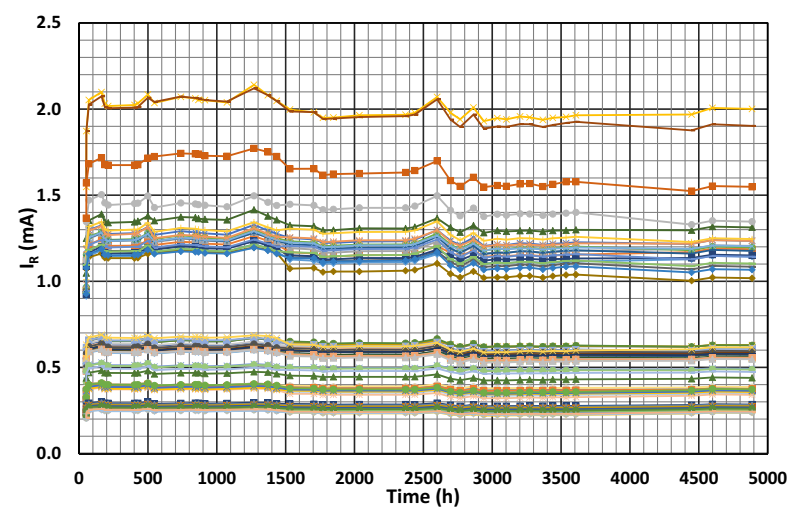

Figure 6. Reverse leakage current evolution

$$
\lambda=\frac{x}{T D H \cdot A F}
$$

where,

- $\lambda$ is the failure rate in number of failures $/ 10^{6}$ hours

- $x$ is the number of failures

- $T D H$ is the summation of the number of units in operation multiplied by the time of operation.

- $A F$ is the acceleration factor

And, additionally, the mean time to failure is defined as:

$$
M T T F=\frac{1}{\lambda}
$$

where,

- $M T T F$ is the mean time to failure (time to fail of $63 \%$ of the population).

- $\lambda$ is the failure rate.

Although, degradation has not been detected and no broken diodes appeared after 4887 hours of test, and the exact value of the failure rate cannot be obtained. Anyhow, is possible to obtain comply with predicted maximum value of the failure rate in case one diode brakes down just one hour after measuring the devices.

Finally, the failure rate can be calculated by using $x=1$, $T D H=80$ devices multiplied by 4887 hours, and $A F=$ 8 (as previously calculated) in Eq. 9:

$$
\lambda<\frac{1}{80 \cdot 4887 \cdot 8}=0.320 / 10^{6}
$$

The mean time to failure (MTTF), in this case, using the equation 10 is:

$$
M T T F>\frac{1}{\lambda}=3127680 \mathrm{~h}
$$




\section{CONCLUSION}

This paper studies the effect of the derating rules, applied to reversed polarized Schottky diodes, to the failure rate with the aim of comparing the derating rules published by ESA or NASA with the experimental results.

A high temperature reverse bias experiment is developed using a population of 80 diodes divided in 4 groups ( 4 different derating values) of 20 diodes each, with the aim of measuring the leakage current of every single diode reverse polarized.

With $4887 \mathrm{~h}$ under test at $100^{\circ} \mathrm{C}$, which is equivalent to more than $39000 \mathrm{~h}$ at $25^{\circ} \mathrm{C}$, no degradation has been detected and no broken diode happened, obtaining a maximum value of the failure rate equal to 0.320 failures $/ 10^{6} \mathrm{~h}$.

The absence of failures in the diodes, after $4887 \mathrm{~h}$ under test at $100{ }^{\circ} \mathrm{C}$ with a derating between $100 \%$ and $30 \%$, means that in short term (taking into account the scope of this work with only $4887 \mathrm{~h}$ under test) the derating has not reduced the failure rate.

This fact points out the derating rules should be reviewed in order to take account the duration of the mission, for instance, the increase of the derating values at least in 6-months missions.

\section{REFERENCES}

[1] ESA - ESTEC, Noordwijk, The Netherlands. Space product assurance: Derating - EEE components, ECSS-Q-ST-30-11C Rev 1, Oct. 42011.

[2] NASA - Goddard Space Flight Center, Greenbelt, Maryland. Instructions for EEE Parts Selection, Screening, Qualification, and Deratings, EEE-INST-002, May 2003.

[3] NASA, Washington DC. Reliability preferred practices for design and test, Memorandum 4322, Sept. 1991.

[4] Richard Denning. Applied R\&M manual for defence system. SARS, MoD, Abbey Wood, May 2012.

[5] Zhaofeng Huang. De-rating design and analysis for safety and reliability. In Reliability and Maintainability Symposium (RAMS), 2014 Annual, pages 1-7, Jan 2014.

[6] Kwok-Wai Ma. Highly reliable dc/dc power module for demanding applications-from design to field tracking. In Applied Power Electronics Conference and Exposition, 1997. APEC'97 Conference Proceedings 1997., Twelfth Annual, volume 2, pages 647-654 vol.2, Feb 1997.

[7] ESA. European preferred parts list, ISSUE 30, Dec. 2015.

[8] G.C. Sikora and L.E. Miller. Application of power step stress techniques to transistor life predictions. In Physics of Failure in Electronics, 1964. Third Annual Symposium on the, pages 30-42, Sept 1964.

[9] N. F. Bechtold and C. L. Hanks. Failure-rate studies on silicon rectifiers. American Institute of Electrical Engineers, Part I: Communication and Electronics, Transactions of the, 77(1):49-56, March 1958.
[10] J.A. McLinn. Creating a sensible derating system. In Reliability and Maintainability Symposium, 2006. RAMS '06. Annual, pages 362-367, Jan 2006.

[11] Department of defense, Washington DC. Military handbook: Reliability prediction of electronic equipment, MIL-HDBK-217F, Dec. 2, 1991. 